Revta brasil. Bot., São Paulo, V.23, n.2, p.121-132, jun. 2000

\title{
Padrões estruturais quantitativos de bancos de Sargassum (Phaeophyta, Fucales) do litoral dos estados do Rio de Janeiro e São Paulo, Brasil ${ }^{1}$
}

\author{
MARIA TERESA M. DE SZÉCHY ${ }^{2,4}$ e ÉDISON J. DE PAULA ${ }^{3}$
}

(recebido em 11 de setembro de 1998; aceito em 27 de dezembro de 1999)

\begin{abstract}
Quantitative structural patterns of Sargassum beds (Phaeophyta, Fucales) of the coasts of Rio de Janeiro and São Paulo states, Brazil). This paper aimed to describe the biological structure of Sargassum beds along the coasts of Rio de Janeiro and São Paulo states. Sixteen rocky shore communities were sampled at random $(50$ x $50 \mathrm{~cm}$ quadrats) during 1991-92. These communities presented populations of distinct Sargassum species. They were chosen because they were subjected to different degrees of wave action and emersion. Seven infrageneric taxa of this genus were studied. The Sargassum dry mass varied from $37 \mathrm{~g} . \mathrm{m}^{-2}$ to $587 \mathrm{~g} . \mathrm{m}^{-2}$; the highest values were found in moderate and protected sites. The cluster analysis of these communities, considering the dry mass of 75 groups of macroalgae and macroinvertebrates, indicated three structural patterns characterized by the most abundant group of organisms: 1) Sargassum, in places not exposed to direct wave action, frequent emersion nor recent disturbances; 2) calcareous algae (Corallinaceae), mainly in exposed places, with Phragmatopoma lapidosa (tube worm) and/ or Perna perna (mussels); 3) other groups of non-calcareous algae, such as Dictyopteris delicatula, representative in intermediate situations. These patterns may be usefull to study the exploitation potentiality of Sargassum and other organisms, like mussels, in the Brazilian coast.
\end{abstract}

RESUMO - (Padrões estruturais quantitativos de bancos de Sargassum (Phaeophyta, Fucales) do litoral dos estados do Rio de Janeiro e São Paulo, Brasil). Este trabalho objetivou descrever a estrututura biológica de bancos de Sargassum do litoral dos estados do Rio de Janeiro e São Paulo. Comunidades de 16 costões rochosos foram amostradas aleatoriamente (quadrados de $50 \mathrm{~cm}$ de lado) durante 1991-92. Essas comunidades, que apresentavam populações de Sargassum de sete diferentes táxons infragenéricos, eram submetidas a diferentes graus de exposição às ondas e à emersão. A massa seca de Sargassum variou de $37 \mathrm{~g} . \mathrm{m}^{-2}$ a 587 g.m $\mathrm{m}^{-2}$; os maiores valores foram encontrados em locais moderados e protegidos. A análise de agrupamento dessas comunidades, considerando a massa seca de 75 grupos de macroalgas e macroinvertebrados, indicou três padrões estruturais caracterizados pelo grupo de organismos mais abundantes: 1) Sargassum, em locais não expostos à ação direta das ondas, nem à emersão frequiente e distúrbios recentes; 2) algas calcárias (Corallinaceae), principalmente em locais expostos à ação das ondas e à emersão, juntamente com Phragmatopoma lapidosa (poliqueto tubícola) e/ou Perna perna (mexilhão); 3) outros grupos de macroalgas não calcárias, como Dictyopteris delicatula, representativos em situações intermediárias. Esses padrões poderão ser úteis para a avaliação do potencial de explotação de Sargassum no litoral brasileiro, bem como de outros organismos como mexilhões.

Key words - Sargassum, Corallinaceae, dry mass, structural patterns

\section{Introdução}

Em regiões tropicais e subtropicais, Sargassum C. Agardh (Phaeophyta, Fucales) pode formar densos bancos (De Wreede 1976, Prince \& O'Neal 1979, Lüning 1990). Em algumas localidades da costa do Brasil, representa a alga mais importante em termos de abundância (Eston et al. 1986, Paula \& Eston 1987, Paula 1989, Eston \& Bussab 1990).

1. Parte da tese de doutorado de M. T. M. Széchy.

2. Departamento de Botânica, Instituto de Biologia, Centro de Ciências da Saúde, Universidade Federal do Rio de Janeiro, Ilha do Fundão, 21941-590 Rio de Janeiro, RJ, Brasil.

3. Departamento de Botânica, Instituto de Biociências, Universidade de São Paulo, Caixa Postal 11461, 05422-970 São Paulo, SP, Brasil.

4. Autor para correspondência: szechy@ biologia. ufrj.br.
Além de fonte alternativa para as indústrias de alginato, como vem acontecendo na Índia (Thomas \& Subbaramaiah 1991), essa macroalga tem sido aproveitada também para fins medicinais (Masuda et al. 1993), como ração para animais domésticos (Trono Jr. \& Lluisma 1990) e como meio de incrementar a produção de pescado em regiões costeiras (Ohno et al. 1990). No entanto, até o momento, os bancos brasileiros de Sargassum não são explotados.

Oliveira Filho \& Paula (1979) estimaram o potencial de coleta de espécies de Sargassum do litoral de São Paulo, com base em fotografias aéreas e em amostras feitas em áreas selecionadas, excluindo do estudo as populações de costões muito expostos às ondas e as localizadas abaixo de $2 \mathrm{~m}$ do limite da maré baixa. Esses autores concluíram que a costa sudeste brasileira teria matéria-prima suficiente para suportar o consumo interno de algina, reconhecendo os bancos de locais protegidos como os mais promis- 
sores. Mais tarde, porém, Oliveira (1990) discordou dessa possibilidade de aproveitamento das espécies de Sargassum das costas tropicais e subtropicais do Atlântico da América do Sul, em função da quantidade insuficiente. Sem dúvida, essa questão permanece aberta a discussões, considerando a lacuna de informações sobre esse recurso.

Para os estados do Rio de Janeiro e São Paulo, no litoral sudeste brasileiro, onde se concentram os estudos sobre esse gênero, são referidas sete espécies (Paula 1988, Széchy \& Cordeiro-Marino 1991). Diferenças estruturais foram descritas para algumas comunidades de Sargassum submetidas a diferentes graus de exposição às ondas e distribuições verticais no costão rochoso. Populações de Sargassum cymosum C. Agardh de locais protegidos mostraram-se mais abundantes em termos de biomassa, apesar de menos densas (menor número de plantas por metro quadrado), quando comparadas a populações de locais expostos (Paula \& Oliveira Filho $1980,1982)$. Foram também documentadas diferenças quanto à composição específica da flora e fauna associadas (Oliveira Filho \& Mayal 1976, Paula \& Oliveira Filho 1980, Oliveira Filho \& Paula 1983). Padrões estruturais para essas comunidades, contudo, não foram estabelecidos.

Este estudo teve por objetivo descrever a estrutura biológica de comunidades de costões rochosos do litoral dos estados do Rio de Janeiro e São Paulo (Brasil), onde ocorriam diferentes espécies de Sargassum, definindo padrões quantitativos com base na massa seca das macroalgas e macroinvertebrados presentes.

\section{Material e métodos}

Dezesseis pontos de coleta foram selecionados na área geográfica compreendida entre os municípios de Peruíbe, sul do estado de São Paulo, e Macaé, norte do estado do Rio de Janeiro $\left(22^{\circ} 27^{\prime}-24^{\circ} 24^{\prime}\right.$ 'S e $\left.41^{\circ} 51^{\prime} 30^{\prime \prime}-47^{\circ} 00^{\prime} 00^{\prime \prime} \mathrm{W}\right)$. As coletas foram realizadas nos anos de 1991 e 1992, preferencialmente na primavera. Seis dos pontos de coleta foram visitados mais de uma vez, perfazendo um total de 26 observações (tabela 1).

Os pontos de coleta foram caracterizados quanto ao grau de exposição às ondas e à posição vertical do banco de Sargassum no costão (tabela 1). A caracterização quanto ao grau de exposição às ondas foi feita indiretamente, com base na sua posição em relação à linha de costa e na presença de acidentes geográficos. Locais protegidos são os de interior de baías ou enseadas, voltados para seu fundo, onde acidentes geográficos, como pontas rochosas e ilhas, criam uma barreira contra ventos ou correntezas fortes. Locais expostos situam-se fora de baías ou enseadas, ou em sua abertura, em posição tal que recebem diretamente o embate das ondas. Situações intermediárias entre as acima descritas caracterizam os locais moderados.

Segundo Paula (1989), Sargassum cresce na região sublitorânea dos costões rochosos. Assim, a posição vertical dos bancos de Sargassum foi definida em períodos de maré de sizígia, durante as coletas, em: a) acima do nível da maré baixa, b) até $1 \mathrm{~m}$ abaixo do nível da maré baixa e c) entre $1 \mathrm{~m}$ e $2 \mathrm{~m}$ abaixo do nível da maré baixa. A posição vertical no costão foi empregada para estimar o grau de emersão a que estariam submetidos os bancos (Oliveira Filho \& Paula 1983). Dessa forma, bancos de Sargassum de costões expostos às ondas ficariam emersos mais freqüentemente e durante mais tempo, enquanto os de costões protegidos estariam sempre submersos, mesmo nas marés mais baixas. Neste caso, as coletas foram efetuadas através de mergulho livre.

Foram efetuadas raspagens, com espátulas metálicas, do substrato delimitado por quadrados de $50 \mathrm{~cm}$ de lado. Em cada amostragem, esse quadrado foi posicionado em cinco pontos marcados aleatoriamente em uma corda de $10 \mathrm{~m}$ de comprimento, estendida ao longo do banco de Sargassum, paralelamente à linha d'água, evitando-se sempre seus limites superior e inferior de distribuição no costão rochoso. O material obtido pelas raspagens, incluindo organismos vivos e material não vivo, como conchas, carapaças, sedimentos e tubos calcários, foi fixado com solução de formalina a $4 \%$ em água do mar. No laboratório, o material sofreu uma triagem preliminar a olho nu, de modo a separar as macroalgas e os macroinvertebrados de diferentes grupos taxonômicos. As macroalgas foram classificadas quanto ao tipo morfológico, com base na altura, morfologias interna e externa do talo (adaptação de Steneck \& Dethier 1994), em: filamentosas, foliáceas, macrófitas corticadas, macrófitas coriáceas, calcárias articuladas e crostosas (tabela 2). Foram desprezados anfípodas e tanaidáceos, apesar de freqüentes, por considerarmos a técnica de coleta empregada imprópria para esses invertebrados. Cada grupo de organismos triado foi lavado em água doce corrente, sobre uma peneira de $1 \mathrm{~mm}$ de abertura de malha, deixado sobre papel absorvente para retirada do excesso de água e levado à estufa a 70-90 ${ }^{\circ} \mathrm{C}$, por um período mínimo de quatro horas, até atingir massa seca constante. Poliquetos tubícolas foram pesados juntamente com seus tubos. As plantas de Sargassum foram cortadas, de modo a separar o apressório das partes eretas. As pesagens foram feitas em balança digital eletrônica, com precisão de $0,01 \mathrm{~g}$.

Posto que não houve homogeneidade das variâncias, foram aplicados testes não paramétricos. O teste de Kruskal-Wallis (resultado não apresentado) apontou a existência de diferenças significativas entre as médias de massa seca $(\mathrm{p}<0,01)$, tanto entre populações de diferentes espécies/variedades de Sargassum, como entre populações situadas em locais com diferentes graus de exposição às ondas. Posteriormente, foi usado o teste de Comparação Múltipla de Postos para se comparar as médias de massa seca, duas a duas $(\mathrm{p}<0,05)($ Zar 1984).

A determinação dos padrões estruturais foi feita a partir da análise de agrupamento das 26 observações (tabela 1) com base em 75 descritores, ou seja, nos grupos taxonômicos de macroalgas e macroinvertebrados encontrados (tabela 2), empregando o coeficiente de Bray-Curtis e WPGMA (Legendre \& Legendre 1983). Foram calculadas, para cada observação, as médias aritméticas das massas secas de cada grupo taxonômico, obtidas a partir dos quadrados raspados. 


\section{Resultados e Discussão}

Os maiores valores de massa seca de Sargassum foram registrados para $S$. vulgare C. Agardh var. vulgare e S. filipendula C. Agardh da Praia Rasa e da Ponta de Pingo d'Água (observações XXV e
XVIII - tabela 3), em locais moderados, no período primavera-verão, enquanto os menores valores foram registrados para as mesmas espécies do Saco de Piraquara de Dentro e da Ponta da Pitanga (observações XXIII, XV e XVI - tabela 3), em locais protegidos, no período outono-inverno. Assim, no

Tabela 1. Pontos de coleta e datas referentes às 26 observações empregadas no estudo. Caracterização quanto ao grau de exposição às ondas e à posição vertical no costão: $\mathrm{S} 0$ = região sublitorânea acima do nível da maré baixa; $\mathrm{S} 1$ = região sublitorânea abaixo do nível da maré baixa, até $1 \mathrm{~m}$ de profundidade; S2 = região sublitorânea abaixo do nível da maré baixa, entre 1-2 m de profundidade.

\begin{tabular}{|c|c|c|c|c|c|}
\hline Observação & $\begin{array}{l}\text { Estado/ } \\
\text { Município }\end{array}$ & Localidade & $\begin{array}{l}\text { Ponto de } \\
\text { coleta }\end{array}$ & $\begin{array}{l}\text { Grau de exposição às } \\
\text { ondas/posição no costão }\end{array}$ & Data de coleta \\
\hline & São Paulo: & & & & \\
\hline I & Peruíbe & Prainha & 1 & exposto/ S0 & set. 91 \\
\hline II & Itanhaém & Praia de Peruíbe & 2 & moderado/ S0 & set. 91 \\
\hline III & São Sebastião & Praia das Cigarras & 3 & moderado/ S0 & set. 91 \\
\hline IV & Caraguatatuba & Praia Martins de Sá & 4 & moderado/ S0 & nov. 91 \\
\hline $\mathrm{V}$ & Ubatuba & Praia de Itaguá & 5 & moderado/ S0 & out. 91 \\
\hline VI & Ubatuba & Praia de Itaguá & 6 & protegido/ S1 & nov. 91 \\
\hline VII & Ubatuba & Praia de Perequeaçu & 7 & moderado/ S0 & nov. 91 \\
\hline VIII & Ubatuba & Ponta da Fortaleza & 8 & protegido/ S1 & out. 91 \\
\hline IX & & & & & abr. 92 \\
\hline $\mathrm{X}$ & Ubatuba & Ponta da Fortaleza & 9 & moderado/ S0 & out. 91 \\
\hline XI & & & & & abr. 92 \\
\hline XII & Ubatuba & Ponta da Fortaleza & 10 & exposto/ S0 & out. 91 \\
\hline XIII & & & & & abr. 92 \\
\hline XIV & $\begin{array}{l}\text { Rio de Janeiro: } \\
\text { Angra dos Reis }\end{array}$ & $\begin{array}{l}\text { Ponta da Pitanga/Saco de } \\
\text { Piraquara de Fora }\end{array}$ & 11 & protegido/ S2 & jan. 92 \\
\hline$X V$ & & & & & jun. 92 \\
\hline XVI & & & & & set. 92 \\
\hline XVII & & & & & nov. 92 \\
\hline XVIII & Angra dos Reis & $\begin{array}{l}\text { Ponta de Pingo d'Água/ } \\
\text { Saco de Piraquara de Fora }\end{array}$ & 12 & moderado/ S0 & jan. 92 \\
\hline XIX & & & & & jun. 92 \\
\hline $\mathrm{XX}$ & & & & & ago. 92 \\
\hline XXI & Angra dos Reis & $\begin{array}{l}\text { Ponta da Fortaleza/Saco } \\
\text { de Piraquara de Dentro }\end{array}$ & 13 & protegido/ S2 & jan. 92 \\
\hline XXII & & & & & jun. 92 \\
\hline XXIII & & & & & ago. 92 \\
\hline XXIV & Mangaratiba & Praia Vermelha/Ibicuí & 14 & moderado/ S0 & out. 92 \\
\hline XXV & Búzios & Praia Rasa & 15 & moderado/ S0 & out. 92 \\
\hline XXVI & Macaé & Praia de Itapebuçu & 16 & moderado/ S0 & out. 92 \\
\hline
\end{tabular}


período considerado, a maior abundância de Sargassum em termos de massa seca deu-se em bancos submetidos a grau moderado de exposição às ondas, posicionados acima do nível da maré baixa, amostrados no período primavera-verão, enquanto que a menor abundância ocorreu em bancos de locais protegidos, posicionados a 1-2 $\mathrm{m}$ de profundidade, amostrados no período outono-inverno.

No entanto, essa tendência nem sempre foi observada, como nas Praias de Peruíbe e Vermelha (observações II e XXIV - tabela 3), onde populações de S. stenophyllum Mart. e S. cymosum C. Agardh var. cymosum mostraram valores de massa seca relativamente baixos, mesmo tratando-se de locais moderados. Em ambos os casos, a interferência do homem no costão, através de atividades como a coleta de mariscos e a poluição da água do mar, era perceptível quando das visitas, apontando para a provável influência destes fatores no estabelecimento e manutenção dos bancos de Sargassum.

A massa seca da população de Sargassum cymosum var. nanum E.J. Paula \& E.C. Oliveira da

Tabela 2. Grupos taxonômicos de macroalgas e de macroinvertebrados empregados na análise de agrupamento. Macroalgas classificadas quanto ao tipo morfológico. * - macroalgas e macroinvertebrados representados no quadrado por poucos indivíduos e agrupados, para pesagem.

\begin{tabular}{|c|c|c|c|}
\hline \multicolumn{4}{|c|}{ Macroalgas } \\
\hline Filamentosas & Foliáceas & Macrófitas corticadas & Macrófitas coriáceas \\
\hline $\begin{array}{l}\text { Asparagopsis taxiformis- } \\
\text { gametófito }\end{array}$ & Cryptopleura ramosa & Acanthophora spicifera & Bryothamnion seaforthii \\
\hline A.taxiformis-tetrasporófito & Dictyopteris delicatula & Champia spp. & Sargassum spp. \\
\hline Bryocladia spp. & D. plagiogramma & Chnoospora minima & \\
\hline Caulerpa ambigua & Dictyota spp. & Chondracanthus spp. & Calcárias articuladas \\
\hline Chaetomorpha spp. & Enteromorpha spp. & Codium spp. & \\
\hline Cladophora spp. & Lobophora variegata & Colpomenia sinuosa & coralináceas articuladas \\
\hline Dasya spp. & Padina spp. & Gastroclonium parvum & Galaxaura spp. \\
\hline Ectocarpales & Rhodymenia spp. & Gelidiopsis spp. & \\
\hline Heterosiphonia spp. & Spatoglossum schroederi & Gelidium spp. & Crostosas \\
\hline Levringia brasiliensis & Ulva spp. & Gracilaria spp. & \\
\hline Polysiphonia spp. & & Grateloupia spp. & crostas calcárias \\
\hline Pterosiphonia spp. & & Gymnogongrus griffithsiae & Peyssonnelia spp. \\
\hline Sphacelaria spp. & & Hypnea spp. & \\
\hline Spyridia spp. & & Laurencia spp. & diatomáceas coloniais \\
\hline \multirow[t]{3}{*}{ não identificadas } & & Plocamium brasiliense & outras algas* \\
\hline & & Pterocladia spp. & \\
\hline & \multicolumn{2}{|c|}{ Macroinvertebrados } & \\
\hline Actiniários & Corais pétreos & Isópodos & Poríferos \\
\hline Aplisiáceos & Crinóides & Nudibrânquios & Sipúnculos \\
\hline Ascidiáceos & Decápodos & Ofiuróides & Turbelários \\
\hline Asteróides & Equinóides & Pantópodos & Zoantídeos \\
\hline Bivalvos & Gastrópodos & Poliplacóforos & Tubos de poliquetos \\
\hline Briozoários & Hidrozoários & Poliquetos & \\
\hline Cirripédios & Holoturóides & & outros animais* \\
\hline
\end{tabular}


Ponta da Fortaleza, face exposta, em Ubatuba (observações XII e XIII - tabela 3) mostrou-se cerca de quatro vezes menor se comparada a estudos anteriores (Paula \& Oliveira Filho 1980, 1982). Esse decréscimo na massa seca pode ter ocorrido também em função de distúrbios naturais ou provocados pelo homem, como a periódica raspagem do costão por pescadores para a retirada de mexilhões. Essa hipótese é respaldada pelos estudos de Chiappone \& Sullivan (1994) e de Hockey \& Bosman (1986).
A variação quantitativa de Sargassum entre os bancos analisados concorda com o referido para outras espécies do gênero (Umezaki 1974, De Wreede 1976, Paula \& Oliveira Filho 1980, 1982, Trono Jr. \& Lluisma 1990, Largo et al. 1994) e destaca a importância da abordagem espaçotemporal, em diferentes escalas, em estudos descritivos de comunidades.

A percentagem de massa seca relativa às partes eretas das plantas correspondeu a mais da metade da

Tabela 3. Massa seca de Sargassum por observação. Total $=$ massa seca total $\left(\mathrm{g} \cdot \mathrm{m}^{-2}\right) ; \%$ Ereta $=$ percentagem da massa seca das partes eretas em relação à massa seca total; $\bar{x}=$ média aritmética; $\mathrm{s}=$ desvio padrão (vide tabela 1 , para a caracterização das observações).

\begin{tabular}{|c|c|c|c|c|}
\hline \multirow[t]{2}{*}{ Observações } & \multirow[t]{2}{*}{ Espécie predominante } & \multicolumn{2}{|c|}{ Total } & \multirow[t]{2}{*}{$\%$ Ereta } \\
\hline & & $\bar{x}$ & $\mathrm{~s}$ & \\
\hline I & S. rigidulum & 100,52 & 86,04 & 70,62 \\
\hline II & S. stenophyllum & 115,50 & 52,15 & 81,32 \\
\hline III & S. stenophyllum & 287,03 & 23,64 & 88,83 \\
\hline IV & S. stenophyllum & 159,63 & 53,56 & 97,03 \\
\hline $\mathrm{V}$ & S. vulgare var. nanum & 332,67 & 58,75 & 72,92 \\
\hline VI & S. vulgare var. vulgare & 213,94 & 82,47 & 96,02 \\
\hline VII & S. cymosum var. cymosum & 277,30 & 64,92 & 94,51 \\
\hline VIII & S. filipendula & 368,39 & 42,44 & 96,81 \\
\hline IX & S. filipendula & 337,24 & 32,46 & 94,95 \\
\hline $\mathrm{X}$ & S. filipendula & 318,47 & 25,92 & 89,88 \\
\hline XI & S. filipendula & 406,77 & 28,78 & 95,07 \\
\hline XII & S. cymosum var. nanum & 102,86 & 41,21 & 53,50 \\
\hline XIII & S. cymosum var. nanum & 105,99 & 35,42 & 53,83 \\
\hline XIV & S. vulgare var. vulgare & 177,09 & 96,27 & 93,42 \\
\hline $\mathrm{XV}$ & S. vulgare var. vulgare & 66,00 & 10,52 & 84,74 \\
\hline XVI & S. vulgare var. vulgare & 68,38 & 13,03 & 78,25 \\
\hline XVII & S. vulgare var. vulgare & 88,02 & 23,67 & 84,85 \\
\hline XVIII & S. filipendula & 414,06 & 72,09 & 95,74 \\
\hline XIX & S. filipendula & 163,45 & 33,66 & 86,74 \\
\hline $\mathrm{XX}$ & S. filipendula & 101,88 & 25,84 & 87,26 \\
\hline XXI & S. filipendula & 366,92 & 50,48 & 95,11 \\
\hline XXII & S. filipendula & 123,18 & 14,34 & 85,56 \\
\hline XXIII & S. filipendula & 36,59 & 9,52 & 71,44 \\
\hline XXIV & S. cymosum var. cymosum & 82,21 & 19,52 & 91,04 \\
\hline XXV & S. vulgare var. vulgare & 586,66 & 56,77 & 85,90 \\
\hline XXVI & S. vulgare var. nanum & 356,34 & 42,88 & 83,77 \\
\hline
\end{tabular}


sua massa seca total, especialmente em populações de locais protegidos e moderados (observações IV, VIII e VI - tabela 3), chegando a alcançar cerca de 97\%. A maior percentagem de massa seca das partes eretas pode ser interessante quando se objetiva o aproveitamento econômico de Sargassum, através de coleta por poda, conforme aconselham Oliveira Filho \& Paula (1979), já que garante um bom rendimento e a posterior recuperação do banco a partir da regeneração dos apressórios não coletados (Norton 1977, Paula \& Eston 1987).

Em locais expostos, os valores comparativamente menores para a percentagem da massa seca das partes eretas (observações XII e XIII - tabela 3) correspondem a plantas relativamente menores $(2,34$ $\pm 0,77 \mathrm{~cm}$ e $1,88 \pm 0,74 \mathrm{~cm}$, respectivamente) e com apressórios bem mais desenvolvidos, o que está de acordo com o descrito para populações de $S$. cymosum da mesma região (Paula \& Oliveira Filho 1982). O menor desenvolvimento vegetativo das plantas de locais expostos pode ser explicado pelo estresse mecânico provocado pelo batimento das ondas e pela exposição ao ar durante as marés baixas (Paula \& Oliveira Filho 1982, De Ruyter van Steveninck \& Breeman 1987, Kilar \& Hanisak 1989).

A massa seca total de Sargassum foi significativamente diferente entre algumas espécies e variedades. Isso ocorreu principalmente com $S$. vulgare var. nanum E. J. Paula e S. filipendula, que foram as mais representativas, atingindo cerca de $345 \mathrm{~g} \cdot \mathrm{m}^{-2} \mathrm{e}$ 259 g. $\mathrm{m}^{-2}$ de valores médios de massa seca total, respectivamente (tabela 4). Esses valores aproximam-se dos referidos anteriormente para o sudeste do Brasil - até 491 g.m ${ }^{-2}$ (Oliveira Filho \& Paula 1979), para Curaçao - até 440 g.m $\mathrm{m}^{-2}$ (Wanders 1976) e para o Havaí - cerca de 330 g.m ${ }^{-2}$ (De Wreede 1976). Entretanto, mostraram-se nitidamente inferiores quando comparados a bancos de outras espécies e de outras regiões tropicais, como Filipinas

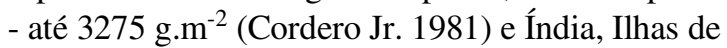
Mandapam e Krusadai - até 2400 g.m ${ }^{-2}$ (Thomas \& Subbaramaiah 1991).

O maior valor de massa seca total encontrado para S. vulgare var. nanum (tabela 4), considerando que a altura média das plantas não foi expressiva $(3,54 \pm 1,88 \mathrm{~cm}$ e $6,81 \pm 2,41 \mathrm{~cm}$, observações $\mathrm{V}$ e XXVI, respectivamente), pode ser associado à alta densidade, particularmente na Praia de Itapebuçu (584 plantas.m ${ }^{-2}$, observação XXVI).

Populações de Sargassum, independentemente da espécie ou variedade, apresentaram maior massa seca total em locais moderados, alcançando, em média, 302 g.m ${ }^{-2}$ (tabela 5). Na literatura, essa tendência também é apontada, se bem que com maior ênfase aos locais protegidos e, em sua maioria, na região sublitorânea rasa (Oliveira Filho \& Paula 1979, Paula \& Oliveira Filho 1982, Umezaki 1984).

Populações de locais expostos e protegidos não mostraram entre si diferenças significativas quanto à massa seca total de Sargassum (tabela 5), apesar da massa seca dos apressórios ter sido significativamente maior em locais expostos (ao nível de 5\%, teste de Comparação Múltipla de Postos), o que concorda com Oliveira Filho \& Paula (1979) e Paula \& Oliveira Filho (1982). A similaridade de massa seca total entre populações localizadas em graus extremos de exposição às ondas pode ser justificada pelo comportamento inverso entre altura das plantas e densidade, seguindo o esperado de acordo com a lei de "self-thinning", como bem o ilustraram Cousens \& Hutchings (1983) e Hara et al. (1986).

Além de Sargassum, algas calcárias da família Corallinaceae foram bastante representativas nas comunidades. De um modo geral, onde Sargassum apresentou seus maiores valores de massa seca, as algas calcárias articuladas mostraram seus menores valores, com exceção da Praia de Itapebuçu (observação XXVI), onde ambas foram abundantes; numerosas plantas pouco desenvolvidas de Sargassum

Tabela 4. Massa seca total $\left(\mathrm{g} \cdot \mathrm{m}^{-2}\right)$ de diferentes espécies e variedades de Sargassum. $\mathrm{n}=$ número de unidades amostrais; $\overline{\mathrm{x}}=$ média aritmética; $\mathrm{s}=$ desvio-padrão. As barras verticais ligam os valores que não diferem entre si, pelo teste de Comparação Múltipla de Postos $(\mathrm{p}<0,05)$.

\begin{tabular}{lrc|r}
\hline Espécies & $\mathrm{n}$ & $\overline{\mathrm{x}}$ & \multicolumn{1}{c}{$\mathrm{s}$} \\
\hline S. rigidulum & 5 & 100,52 & 96,20 \\
S. cymosum var. nanum & 10 & 104,44 & 40,52 \\
S. stenophyllum & 15 & 187,40 & 88,68 \\
S. cymosum var. cymosum & 9 & 190,60 & 115,76 \\
S. vulgare var. vulgare & 30 & 200,00 & 193,96 \\
S. filipendula & 48 & 259,40 & 141,96 \\
S. vulgare var. nanum & 10 & 344,52 & 55,64 \\
\hline
\end{tabular}


cresciam sobre um tapete de calcárias articuladas. A massa seca de calcárias articuladas variou de desprezível (< 0,01 g.m ${ }^{-2}$ ), na Praia das Cigarras (observação III) a 369,75 g.m ${ }^{-2}$, na Praia de Itapebuçu (observação XXVI), podendo ser abundantes em locais expostos, moderados ou protegidos.

A abundância da família Corallinaceae em bancos de Sargassum foi também documentada por Oliveira Filho \& Mayal (1976), Eston et al. (1986), Yoneshigue \& Valentin (1988) e Fonseca (1998), para o litoral brasileiro, e por Hoek (1969) e Kimura et al. (1987), para as costas das Bahamas, Curaçao e Japão, sugerindo um processo de competição interespecífica. Fonseca (1998) indica a competição como o principal mecanismo estruturador de comunidades de $S$. furcatum Kütz. da região sublitorânea da Praia do Forno, em Búzios, Rio de Janeiro.

Outras macroalgas, de tipos morfológicos diferentes, foram representativas em termos de massa seca em uma ou outra das comunidades estudadas. As filamentosas, incluindo principalmente Bryocladia thyrsigera (J. Agardh) F. Schmitz in Falkenb. (59,09 g.m ${ }^{-2}$ ) e Pterosiphonia spp. $\left(2,00 \mathrm{~g} . \mathrm{m}^{-2}\right)$, foram abundantes na Praia de Peruíbe (observação II), onde ficavam parcialmente soterradas pela areia. Chaetomorpha antennina (Bory) Kütz., outra filamentosa, mostrou-se mais abundante $\left(1,28 \mathrm{~g} . \mathrm{m}^{-2}\right)$ na Prainha (observação I), em locais de forte arrebentação das ondas. Dentre as foliáceas, Ulva spp. foram mais abundantes $\left(8,23 \mathrm{~g} . \mathrm{m}^{-2}\right)$ na Praia de Perequeaçu (observação VII), local eutrofizado por esgotos domésticos, enquanto Dictyopteris delicatula J. V. Lamour. foi mais abundante $\left(42,40\right.$ g.m $\left.{ }^{-2}\right)$ na Praia Vermelha (observação XXIV). As macrófitas corticadas, especialmente Laurencia spp. (27,05 g. $\left.\mathrm{m}^{-2}\right)$ e Chondracanthus spp.

Tabela 5. Massa seca total (g.m $\left.\mathrm{m}^{-2}\right)$ de Sargassum de localidades com diferentes graus de exposição às ondas. $\mathrm{n}=$ número de unidades amostrais; $\overline{\mathrm{x}}=$ média aritmética; $\mathrm{s}=$ desvio padrão. A barra vertical liga os valores que não diferem entre si, pelo teste de Comparação Múltipla de Postos ( $<<0,05)$.

\begin{tabular}{lccr}
\hline Grau de exposição às ondas & $\mathrm{n}$ & $\overline{\mathrm{x}}$ & \multicolumn{1}{c}{$\mathrm{s}$} \\
\hline Exposto & 15 & 103,12 & 60,84 \\
Protegido & 48 & 176,96 & 131,08 \\
Moderado & 64 & 302,08 & 147,08 \\
\hline
\end{tabular}

(20,98 g.m $\left.{ }^{-2}\right)$, alcançaram seus valores máximos de massa seca na Ponta de Pingo d'Água (observação XVIII) e na Praia de Perequeaçu (observação VII), respectivamente. Algas crostosas calcárias alcançaram seu máximo valor de massa seca $\left(220,49 \mathrm{~g} \cdot \mathrm{m}^{-2}\right)$ na Prainha (observação I).

Em relação aos macroinvertebrados, poliquetos, especialmente Phragmatopoma lapidosa Kinberg $\left(897,31\right.$ g.m $\left.{ }^{-2}\right)$, cirripédios $\left(112,21\right.$ g.m $\left.{ }^{-2}\right)$, decápodos $\left(15,22\right.$ g.m ${ }^{-2}$ ) (observação I), equinóides $\left(40,27\right.$ g.m $\left.{ }^{-2}\right)$, isópodos $\left(0,45\right.$ g.m ${ }^{-2}$ ) (observação XII), bivalvos, especialmente Perna perna (Linnaeus) $\left(426,93 \mathrm{~g} \cdot \mathrm{m}^{-2}\right)$ e poliplacóforos $\left(0,30 \mathrm{~g} \cdot \mathrm{m}^{-2}\right)$ (observação XIII) alcançaram seus maiores valores de massa seca em locais expostos. Ascidiáceos $\left(21,15 \mathrm{~g} \cdot \mathrm{m}^{-2}\right)$ (observação XV), gastrópodos $\left(55,54 \mathrm{~g} \cdot \mathrm{m}^{-2}\right)$ (observação XVII), poríferos $\left(29,52 \mathrm{~g} \cdot \mathrm{m}^{-2}\right)$ (observação XIX), zoantídeos $\left(7,67 \mathrm{~g} \cdot \mathrm{m}^{-2}\right)$ e actiniários $\left(0,20 \mathrm{~g} \cdot \mathrm{m}^{-2}\right)$ (observação $\mathrm{XX}$ ) apresentaram valores máximos em locais moderados ou protegidos, particularmente da região de Angra dos Reis. Hidrozoários $\left(9,74\right.$ g.m $\left.\mathrm{m}^{-2}\right)$ e briozoários $\left(2,49 \mathrm{~g} \cdot \mathrm{m}^{-2}\right)$ foram mais abundantes em locais moderados (observações XI e II, respectivamente).

A maior abundância de $P$. perna em locais expostos concorda com outros estudos realizados no Brasil (Oliveira Filho \& Mayal 1976), África do Sul (Lambert \& Steinke 1986), Ghana (Evans et al. 1993) e Namíbia (Engledow \& Bolton 1994). $P$. lapidosa também é citado mais frequientemente para locais expostos, onde as condições são mais favoráveis à sua alimentação e à construção de seus tubos (Narchi \& Rodrigues 1965).

A análise de agrupamento das 26 observações revela a formação de dois agrupamentos maiores, que correspondem aos padrões estruturais denominados de A e B. O padrão B pode ser sub-dividido em dois agrupamentos menores: $\mathrm{B}_{1}$ e $\mathrm{B}_{2}$ (figura 1).

As observações classificadas no padrão $\mathrm{B}_{1}$ mostraram-se as mais similares entre si, enquanto que as do padrão $\mathrm{A}$, as menos similares entre si. $\mathrm{O}$ padrão $\mathrm{B}_{1}$ foi o mais bem representado, ocorrendo em 10 observações. A observação XXIII mostrou fraca relação com as demais, enquanto que a observação XXIV apresentou uma relação fraca com o padrão $\mathrm{B}_{2}$.

Algumas observações referentes ao mesmo ponto de coleta, mas de épocas do ano distintas, 
foram classificadas em padrões estruturais diferentes, especialmente as da região de Angra dos Reis, como Ponta de Pingo d'Água: XIX - outono e XX inverno (padrão $B_{2}$ ) ficaram separadas de XVIII verão (padrão $B_{1}$ ) (figura 1 ).

Os padrões estruturais podem ser diferenciados em função dos organismos mais importantes em termos de massa seca: macroalgas de tipos morfológicos diferentes, principalmente Sargassum e calcárias articuladas, e macroinvertebrados sésseis filtradores, como poliquetos tubícolas e bivalvos (tabela 6). Considerando-se o coeficiente de variação da massa seca das variáveis acima, Sargassum mostrou-se a menos heterogênea, entre observações classificadas em um mesmo padrão estrutural. Algas calcárias articuladas, bivalvos e poliquetos tubícolas mostraram-se mais ou menos heterogêneos, de acordo com o padrão estrutural (tabela 6).

Chave artificial para diferenciação dos bancos de Sargassum quanto ao padrão estrutural quantitativo:

1. Bancos com algas calcárias (Corallinaceae) abundantes e, em sua maioria, epilíticas

Padrão A

1. Bancos com algas calcárias (Corallinaceae), porém não abundantes, sendo as articuladas geralmente epífitas Padrão B

2. Sargassum abundante, dominante nos ban$\cos$ Padrão $\mathrm{B}_{1}$

2. Sargassum não abundante; macroalgas de outros tipos morfológicos representativas em termos de massa seca........

Padrão $B_{2}$

Para a caracterização dos padrões estruturais, as espécies mais freqüentes de macroalgas e de alguns grupos de macroinvertebrados serão fornecidas, entre colchetes.

Padrão A - este padrão foi observado em bancos de $S$. cymosum var. nanum, $S$. vulgare var. nanum, $S$. rigidulum Kütz. e S. stenophyllum de locais expostos e moderados, sempre na região sublitorânea acima do nível da maré baixa, na primavera e outono. Estes locais, em sua maioria, sofrem deposição de areia sobre o costão rochoso e também apresentam indícios da ação de raspagem por pescadores, para a coleta de mariscos.
Algas calcárias articuladas da família Corallinaceae [Amphiroa beauvoisii J. V. Lamour., Arthrocardia flabellata (Kütz.) Manza, Corallina panizzoi Schnetter et U. Richt. e Jania adhaerens J. V. Lamour.] alcançaram seus maiores valores médios de massa seca neste padrão estrutural $\left(173,59\right.$ g.m $\left.{ }^{-2}\right)$. O mesmo ocorreu para as crostas calcárias $\left(91,11\right.$ g.m $\left.{ }^{-2}\right)$ (tabela 6$)$.

Sargassum $\left(100,52\right.$ g.m $\mathrm{m}^{-2}<\mathrm{x}<356,34$ g.m $\left.\mathrm{m}^{-2}\right)$ ocorreu em quantidades variáveis.

As filamentosas, especialmente Bryocladia thyrsigera, Sphacelaria spp., Pterosiphonia spp. e Chaetomorpha antennina, foram mais representativas em termos de massa seca neste padrão estrutural. Chnoospora minima (K. Hering) Papenf. $\left(1,33\right.$ g. $\left.\mathrm{m}^{-2}\right)$ também alcançou seus valores máximos de massa seca neste padrão estrutural.

O poliqueto tubícola $P$. lapidosa e bivalvos Modiolus carvalhoi Klappenbach, P. perna, Pteria hirundo (Linnaeus) e Sphenia antillensis Dall et Simpson] foram muito abundantes, alcançando seus maiores valores médios de massa seca (tabela 6). No Ponto IV, Gregariella coralliophaga (Gmelin) substituiu $P$. perna como o bivalvo mais abundante na comunidade. Cirripédios Megabalanus coccopoma (Darwin) e Tetraclita stalactifera (Lamarck), decápodos Pachycheles haigae Rodrigues da Costa, Acanthonyx petiverii $\mathrm{H}$. Milne Edwards e Epialtus brasiliensis Dana, isópodos Cymodoce brasiliensis Richardson e Dynoides castroi Loyola et Silva,

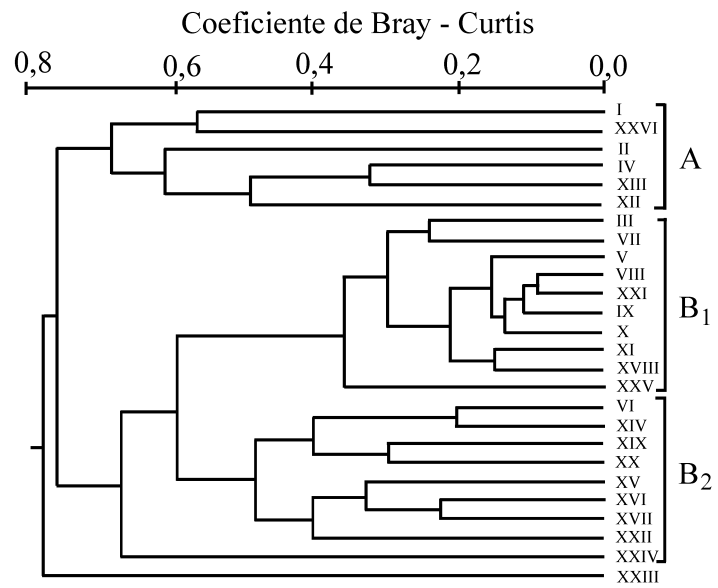

Figura 1. Análise de agrupamento das comunidades estudadas em função da massa seca de macroalgas e macroinvertebrados (26 observações x 75 descritores).

$\mathrm{r}($ valor cofenético $)=0,88$. 
Tabela 6. Média aritmética ( $\overline{\mathrm{x}}$, em g.m $\mathrm{m}^{-2}$ ) e coeficiente de variação (cv, em \%) da massa seca das macroalgas e dos macroinvertebrados mais representativos nos padrões estruturais $\mathrm{A}, \mathrm{B}_{1}$ e $\mathrm{B}_{2}$ e na observação XXIII ( $\mathrm{cv}=$ desvio padrão/ média aritmética x 100).

\begin{tabular}{|c|c|c|c|c|c|c|c|}
\hline & \multicolumn{2}{|c|}{ Padrão A } & \multicolumn{2}{|c|}{ Padrão B 1} & \multicolumn{2}{|c|}{ Padrão $\mathrm{B}_{2}$} & \multirow{3}{*}{ XXIII } \\
\hline & $\overline{\mathrm{x}}$ & $\mathrm{cv}$ & $\overline{\mathrm{x}}$ & $\mathrm{cv}$ & $\overline{\mathrm{x}}$ & $\mathrm{cv}$ & \\
\hline & \multicolumn{2}{|c|}{$(n=6)$} & \multicolumn{2}{|c|}{$(\mathrm{n}=10)$} & \multicolumn{2}{|c|}{$(\mathrm{n}=9)$} & \\
\hline \multicolumn{8}{|l|}{ Macroalgas } \\
\hline Acanthophora spicifera & 0 & 0 & 0,01 & 300 & 0,16 & 163 & 0 \\
\hline Bryocladia thyrsigera & 18,35 & 222 & 0 & 0 & 0 & 0 & 0 \\
\hline Bryothamnion seaforthii & 0 & 0 & 0,84 & 292 & 1,74 & 265 & 0 \\
\hline calcárias articuladas & 173,59 & 113 & 4,72 & 130 & 23,20 & 142 & 229,16 \\
\hline Chaetomorpha antennina & 0,45 & 184 & 0 & 0 & 0 & 0 & 0 \\
\hline Chnoospora minima & 0,64 & 108 & 0 & 0 & 0 & 0 & 0 \\
\hline Chondracanthus spp. & 2,60 & 183 & 6,30 & 233 & 0,10 & 210 & 0,02 \\
\hline Colpomenia sinuosa & 1,67 & 145 & 0,14 & 129 & 0 & 0 & 0 \\
\hline crostas calcárias & 91,11 & 142 & 6,77 & 138 & 0,38 & 153 & 0,01 \\
\hline Dictyopteris delicatula & 8,62 & 111 & 7,24 & 98 & 17,52 & 142 & 3,04 \\
\hline D. plagiogramma & 0 & 0 & 0,07 & 314 & 0,08 & 275 & 0,01 \\
\hline Dictyota spp. & 1,93 & 206 & 0,45 & 202 & 0,51 & 157 & 1,84 \\
\hline Galaxaura spp. & 9,48 & 224 & 2,86 & 210 & 1,69 & 138 & 0 \\
\hline Gelidiopsis spp. & 6,53 & 223 & 3,04 & 162 & 0,97 & 79 & 0,01 \\
\hline Grateloupia spp. & 0,05 & 220 & 0,14 & 307 & 0 & 0 & 0 \\
\hline Hурпеа spp. & 3,42 & 149 & 5,02 & 169 & 1,31 & 95 & 0,17 \\
\hline Laurencia spp. & 0,04 & 250 & 11,71 & 131 & 2,41 & 154 & 1,50 \\
\hline Padina spp. & 0,03 & 133 & 0,58 & 152 & 4,01 & 185 & 0,36 \\
\hline Pterosiphonia spp. & 0,72 & 178 & 0,01 & 400 & 0 & 0 & 0 \\
\hline Rhodymenia spp. & 0,07 & 229 & 1,34 & 287 & 0,02 & 300 & 0 \\
\hline Sargassum spp. & 156,81 & 58 & 369,55 & 23 & 126,97 & 49 & 36,59 \\
\hline Sphacelaria spp. & 0,96 & 222 & 0,14 & 214 & 0 & 0 & 0,01 \\
\hline Ulva spp. & 4,33 & 80 & 2,68 & 207 & 0 & 0 & 0 \\
\hline \multicolumn{8}{|l|}{ Macroinvertebrados } \\
\hline actiniários & 0 & 0 & 0,03 & 233 & 0,08 & 150 & 0,01 \\
\hline ascidiáceos & 0,08 & 213 & 1,00 & 213 & 13,30 & 59 & 13,78 \\
\hline bivalvos & 199,75 & 114 & 6,16 & 118 & 2,32 & 133 & 0,82 \\
\hline cirripédios & 38,57 & 191 & 2,57 & 300 & 0 & 0 & 0 \\
\hline decápodos & 6,77 & 125 & 1,75 & 91 & 1,80 & 140 & 1,05 \\
\hline equinóides & 15,07 & 167 & 0,92 & 166 & 1,18 & 116 & 1,84 \\
\hline gastrópodos & 28,39 & 50 & 23,12 & 34 & 36,30 & 53 & 28,90 \\
\hline isópodos & 0,22 & 105 & 0,03 & 200 & 0 & 0 & 0,02 \\
\hline ofiuróides & 0,02 & 250 & 0,12 & 258 & 0,08 & 138 & 0,02 \\
\hline poliplacóforos & 0,13 & 131 & 0,03 & 200 & 0,06 & 0,08 & 0,02 \\
\hline $\begin{array}{l}\text { poliquetos tubícolas } \\
\text { (indivíduos + tubos) }\end{array}$ & 472,69 & 90 & 0,16 & 201 & 0 & 0 & 0 \\
\hline poríferos & 0,27 & 170 & 10,06 & 127 & 11,44 & 158 & 7,75 \\
\hline zoantídeos & 0 & 0 & 0,30 & 150 & 3,36 & 128 & 0,90 \\
\hline
\end{tabular}


poliplacóforos Ischnochiton sp. e equinóides Echinometra lucunter (Linnaeus) e Paracentrotus gaimardi (Blainville) também foram mais representativos em termos de massa seca neste padrão estrutural (tabela 6).

Os gastrópodos Fissurella spp. estiveram presentes em todas as observações.

Padrão $B_{1}$ - este padrão foi observado em bancos de $S$. cymosum var. cymosum, $S$. vulgare var. vulgare, $S$. vulgare var. nanum, $S$. filipendula e $S$. stenophyllum de locais protegidos e moderados, tanto acima como abaixo do nível da maré baixa, na primavera, outono e verão.

Sargassum ocorreu em grande quantidade $\left(277,30\right.$ g. $\left.\mathrm{m}^{-2}<\mathrm{x}<586,66 \mathrm{~g} \cdot \mathrm{m}^{-2}\right)$, alcançando seu maior valor médio de massa seca neste padrão estrutural (tabela 6), onde formava bancos geralmente densos e homogêneos quanto à fisionomia.

Algas calcárias articuladas [Amphiroa brasiliana Decne, A. fragilissima (L.) J.V. Lamour., Jania adhaerens e J. capillacea Harv.] (4,72 g.m $\left.{ }^{-2}\right)$ e crostosas $\left(6,77 \mathrm{~g} . \mathrm{m}^{-2}\right)$ estiveram presentes, porém não abundantes (tabela 6).

As macrófitas corticadas, como Laurencia spp., Chondracanthus spp. e Hypnea spp., alcançaram seus maiores valores médios de massa seca neste padrão (tabela 6).

Hidrozoários Aglaophenia latecarinata Allman, Sertularia distans (Lamouroux) e $S$. turbinata (Lamouroux) (9,74 g.m $\left.{ }^{-2}\right)$, ofiuróides Ophiactis savignyi (Müller et Troschel) $\left(0,43\right.$ g. $\left.\mathrm{m}^{-2}\right)$, sipúnculos $\left(0,26\right.$ g.m $\left.{ }^{-2}\right)$ e pantópodos Anoplodactylus stictus Marcus e Tanystylum isabellae Marcus (0,04 g. $\left.\mathrm{m}^{-2}\right)$ alcançaram seus valores máximos de massa seca neste padrão estrutural.

Poliquetos tubícolas estiveram geralmente ausentes ou, se presentes, pouco abundantes, não chegando a formar manchas sobre o substrato rochoso $\left(0<\mathrm{x}<0,48 \mathrm{~g} \cdot \mathrm{m}^{-2}\right)$.

Padrão $\mathrm{B}_{2}$ - este padrão foi observado em bancos de $S$. vulgare var. vulgare e $S$. filipendula de locais protegidos e moderados, tanto acima como abaixo do nível da maré baixa, na primavera, verão, outono e inverno. Dentre estes locais, estão costões muito inclinados, sombreados pela proximidade da vegetação terrestre ou com muitos sedimentos finos depositados. Também foi observada a presença marcante de macroherbívoros, como ouriços grandes, peixes e tartarugas.

Sargassum foi menos abundante $\left(66,00 \mathrm{~g} . \mathrm{m}^{-2}\right.$ $<\mathrm{x}<213$,94 g.m ${ }^{-2}$ ), formando bancos mais heterogêneos quanto à físionomia, em função da presença de outras macroalgas que cresciam como epífitas ou sobre o substrato rochoso, nos espaços entre as plantas maiores.

Algas calcárias articuladas (Corallinaceae) estiveram sempre presentes, variando de quantidade $\left(9,74\right.$ g. $\mathrm{m}^{-2}<\mathrm{x}<56,14$ g.m $\left.\mathrm{m}^{-2}\right)$. Dentre estas, Amphiroa brasiliana, A. fragilissima e Jania adhaerens, preferencialmente epilíticas, e J. capillacea, preferencialmente epífita, foram as mais frequientes. Algas calcárias crostosas foram pouco abundantes (tabela 6).

Foliáceas, como um todo, foram mais representativas neste padrão estrutural $\left(78,27\right.$ g.m $\left.{ }^{-2}\right)$. Dentre estas, Dictyopteris delicatula e Padina spp. alcançaram seus maiores valores médios de massa seca neste padrão (tabela 6).

Asparagopsis taxiformis (Delile) Trevis. - fase gametofítica (0,57 g.m $\left.{ }^{-2}\right)$, Acanthophora spicifera (Vahl) Boergesen (0,42 g.m $\left.{ }^{-2}\right)$, Champia spp. $\left(0,19\right.$ g.m $\left.\mathrm{m}^{-2}\right)$ e Dasya spp. $\left(0,03\right.$ g.m $\left.{ }^{-2}\right)$ alcançaram seus valores máximos de massa seca, apesar de pouco expressivos.

Dentre os macroinvertebrados, gastrópodos Tricolia affinis (C.B. Adams) e Mitrella argus (Orbigny), ascidiáceos Herdmannia momus (Savigny), poríferos, zoantídeos e actiniários Anemonia sargassensis Hargitt alcançaram seus maiores valores médios de massa seca neste padrão (tabela 6).

Poliquetos tubícolas não ocorreram sobre o substrato rochoso.

Em síntese, a relação de abundância entre Sargassum e outros membros da macroflora e da macrofauna pode variar de modo significativo, em comunidades bentônicas de costões rochosos do litoral sudeste do Brasil. Sargassum pode ser ou não o componente dominante na comunidade. A variação na estrutura quantitativa dos bancos de Sargassum pode ser relacionada ao grau de exposição às ondas do costão rochoso bem como à posição vertical do banco neste costão, o que influencia, dentre outros aspectos, o grau de emersão das plantas durante as marés baixas. A influência do grau de exposição às ondas, juntamente com o grau de emersão, no padrão estrutural das comunidades de costões rochosos confirma o resultado de estudos an- 
teriores (Paula \& Oliveira Filho 1982, Lambert \& Steinke 1986, Menge \& Sutherland 1987). Sargassum tende a alcançar altos valores de biomassa em comunidades de locais protegidos e moderados, onde não tenha havido distúrbios recentes, o que indica sua potencialidade como recurso explotável. Em comunidades de locais expostos, animais sésseis filtradores, como Phragmatopoma lapidosa e Perna perna, entre outros, tendem a ser os dominantes na etapa final de sucessão, concordando com o descrito por Lambert \& Steinke (1986) e Evans et al. (1993).

Dentre os distúrbios que parecem alterar a estrutura dessas comunidades, são sugeridas a presença de herbívoros em grande quantidade e de sedimentos finos sobre o substrato rochoso e a ação do homem, através da raspagem do costão para coleta de mariscos. Nesses casos, outros organismos, como algas calcárias ou algas de tipos morfológicos mais simples, tendem a dominar, definindo outros padrões estruturais. A relação entre a dominância de algas calcárias na comunidade e a existência de distúrbios é ressaltada por Lambert \& Steinke (1986), Dye (1992) e Steneck \& Dethier (1994). Por outro lado, a presença em bancos de Sargassum de algas foliáceas, como Dictyopteris delicatula, após raspagem, é descrita por Eston \& Bussab (1990) e Fonseca (1998) como um estádio da sucessão nesses bancos.

Deve-se considerar que as comunidades estudadas para definição dos padrões estruturais são dinâmicas, sofrendo variações no espaço e no tempo. Assim sendo, não se deve esperar que seu padrão estrutural seja imutável.

Seguindo as recomendações de Underwood et al. (1991), os padrões estruturais ora definidos devem ser ratificados por estudos mais abrangentes e prolongados. Estudos experimentais devem avaliar o papel de diferentes fatores no processo de sucessão em comunidades da região sublitorânea do litoral sudeste brasileiro.

Agradecimentos - A Sônia B. da Cunha, do Laboratório de Estatística da Universidade Federal do Rio de Janeiro, pela contribuição no tratamento estatístico dos dados. A Sérgio de A. Rodrigues, Yocie Yoneshigue-Valentin, Ricardo Coutinho, Diclá P. Santos, pelas valiosas sugestões e críticas.

\section{Referências bibliográficas}

CHIAPPONE, M. \& SULLIVAN, K.M. 1994. Ecological structure and dynamics of nearshore hard-bottom communities in the Florida Keys. Bulletin of Marine Science 54:747-756.
CORDERO-JR., P.A. 1981. Eco-morphological observation of the genus Sargassum in Central Philippines, including notes on their biomass and bed determination. In Proceedings of the $4^{\text {th }}$ International Coral Reef Symposium. Paragon Printing Corporation, Quezon, p.399-409.

COUSENS, R. \& HUTCHINGS, M.J. 1983. The relationship between density and mean frond weight in monospecific seaweed stands. Nature 301:240-241.

DE RUYTER VAN STEVENINCK, E.D. \& BREEMAN, A.M. 1987. Population dynamics of a tropical intertidal and deepwater population of Sargassum polyceratium (Phaeophyceae). Aquatic Botany 29:139-156.

DE WREEDE, R.E. 1976. The phenology of three species of Sargassum (Sargassaceae, Phaeophyta) in Hawaii. Phycologia 15:175-183.

DYE, A.H. 1992. Experimental studies of succession and stability in rocky intertidal communities subject to artisinal shellfish gathering. Netherlands Journal of Sea Research 30:209-217.

ENGLEDOW, H.R. \& BOLTON, J.J. 1994. Seaweed - diversity within the lower eulittoral zone in Namibia: the effects of wave action, sand inundation, mussels and limpets. Botanica Marina 37:267-276.

ESTON, V.R. \& BUSSAB, W.O. 1990. An experimental analysis of ecological dominance in a rocky subtidal macroalgal community. Journal of Experimental Marine Biology and Ecology 136:170-195.

ESTON, V.R., MIGOTTO, A.E., OLIVEIRA FILHO, E.C., RODRIGUES, S.A. \& FREITAS, J.C. 1986. Vertical distribution of benthic marine organisms on rocky coasts of the Fernando de Noronha Archipelago (Brazil). Boletim do Instituto Oceanográfico de São Paulo 34:37-53.

EVANS, S.M., GILL, M.E., HARDY, F.G. \& SEKU, F.O.K. 1993. Evidence of change in some rocky shore communities on the coast of Ghana. Journal of Experimental Marine Biology and Ecology 172:129-141.

FONSECA, A.C. 1998. Estudos sucessionais em uma comunidade dominada por Sargassum furcatum Kutzing, na região de Búzios, RJ. Dissertação de mestrado, Universidade Federal Fluminense, Niterói.

HARA, T., KOIKE, F. \& MATSUI, K. 1986. Crowding effect in marine macrophytic populations. Botanical Magazine 99:319-321.

HOCKEY, P.A.R. \& BOSMAN, A.L. 1986. Man as an intertidal predator in Transkei: disturbance, community convergence and management of a natural food resource. Oikos 46:3-14.

HOEK, C. VAN DEN. 1969. Algal vegetation-types along the open coasts of Curaçao, Netherlands Antilles, I. Koninklijke Nederlandse Akademie van Wetenschappen Proceedings, series C, 72:537-558.

KILAR, J.A. \& HANISAK, M.D. 1989. Phenotypic variability in Sargassum polyceratium (Fucales, Phaeophyta). Phycologia 28:491-500.

KIMURA, T., OROSCO, C.A. \& OHNO, M. 1987. Ecological study of Sargassum okamurae Yoshida et T. Konno in Tosa Bay, Japan. Reports of the USA Marine Biological Institute of Kochi University 9:149-167.

LAMBERT, G. \& STEINKE, T.D. 1986. Effects of destroying juxtaposed mussel-dominated and coralline algal communities at Umdoni Park, Natal coast, South Africa. South African Journal of Marine Science 4:203-217. 
LARGO, D.B., OHNO, M. \& CRITCHLEY, A.T. 1994. Seasonal changes in the growth and reproduction of Sargassum polycystum C. Ag. and Sargassum siliquosum J. Ag. (Sargassaceae, Fucales) from Liloan, Cebu, in Central Philippines. Japanese Journal of Phycology 42:53-61.

LEGENDRE, L. \& LEGENDRE, P. 1983. Numerical ecology. Elsevier, Amsterdam.

LÜNING, K. 1990. Seaweeds: their environment, biogeography and ecophysiology. John Wiley, New York.

MASUDA, M., AJISAKA, T., KAWAGUCHI, S., QUANG, H. \& DINH, N.H. 1993. The use of Sargassum mcclurei as medical tea in Vietnan. Japanese Journal of Phycology 41:39-42.

MENGE, B.A. \& SUTHERLAND, J.P. 1987. Community regulation: variation in disturbance, competition, and predation in relation to environmental stress and recruitment. The American Naturalist 130:730-757.

NARCHI, W. \& RODRIGUES, S.A. 1965. Observações ecológicas sobre Phragmatopoma lapidosa Kinberg. Ciência e Cultura 17:228.

NORTON, T.A. 1977. Ecological experiments with Sargassum muticum. Journal of the Marine Biological Association of the United Kingdom 57:33-43.

OHNO, M., ARAI, S. \& WATANABE, M. 1990. Seaweed succession on artificial reefs on different bottom substrata. Journal of Applied Phycology 2:327-332.

OLIVEIRA, E.C. 1990. The rationale for seaweed cultivation in South America. In Cultivation of seaweeds in Latin America. (E.C. Oliveira Filho \& N. Kautsky, eds.). Universidade de São Paulo, Coordenadoria de Comunicação Social., São Paulo, p.135-141.

OLIVEIRA FILHO, E.C. \& MAYAL, E.M. 1976. Seasonal distribution of intertidal organisms at Ubatuba, São Paulo (Brazil). Revista Brasileira de Biologia 36: 305-316.

OLIVEIRA FILHO, E.C. \& PAULA, E.J. 1979. Potentiality for algin production in the São Paulo (Brazil) littoral. In Proceedings of the $9^{\text {th }}$ International Seaweed Symposium (A. Jensen \& J. R. Stein, eds.). Science Press, Princeton, p.479-486.

OLIVEIRA FILHO, E.C. \& PAULA, E.J. 1983. Aspectos da distribuição vertical e variação sazonal de comunidades da zona das marés em costões rochosos do litoral norte do Estado de São Paulo. Publicações do Instituto de Pesquisas da Marinha 147:44-71.

PAULA, E.J. 1988. O gênero Sargassum C. Ag. (Phaeophyta Fucales) no litoral do Estado de São Paulo, Brasil. Boletim de Botânica 10:65-118.

PAULA, E.J. 1989. Zonação nos costões rochosos: região entremarés. In Anais do $1^{\circ}$ Simpósio de Ecossistemas da Costa Sul-Sudeste Brasileira: síntese dos conhecimentos. Academia de Ciências do Estado de São Paulo, São Paulo, v.1, p.266-288.
PAULA, E.J. \& ESTON, V.R. 1987. Are there other Sargassum species potentially as invasive as $S$. muticum? Botanica Marina 30:405-410.

PAULA, E.J. \& OLIVEIRA FILHO, E.C. 1980. Phenology of two populations of Sargassum cymosum (Phaeophyta- Fucales) of São Paulo State coast, Brazil. Boletim de Botânica 8:2139.

PAULA, E.J. \& OLIVEIRA FILHO, E.C. 1982. Wave exposure and ecotypical differentiation in Sargassum cymosum (Phaeophyta - Fucales). Phycologia 21:145-153.

PRINCE, J.S. \& O'NEAL, S.W. 1979. The ecology of Sargassum pteropleuron Grunow (Phaeophyceae, Fucales) in the waters off South Florida. I - Growth, reproduction and population structure. Phycologia 18:109-114.

STENECK, R.S. \& DETHIER, M.N. 1994. A functional group approach to the structure of algal dominated communities. Oikos 69:476-498.

SZÉCHY, M.T.M. \& CORDEIRO-MARINO, M. 1991. Feofíceas do litoral norte do Estado do Rio de Janeiro, Brasil. Hoehnea 18:205-241.

THOMAS, P.C. \& SUBBARAMAIAH, K. 1991. Seasonal variations in growth, reproduction, alginic acid, mannitol, iodine and ash contents of brown alga Sargassum wightii. Indian Journal of Marine Sciences 20:169-175.

TRONO JR., G.C. \& LLUISMA, A.O. 1990. Seasonality of standing crop of a Sargassum (Fucales, Phaeophyta) bed in Bolinao, Pangasinan, Philippines. Hydrobiologia 204/205:331-338.

UMEZAKI, I. 1974. Ecological studies of Sargassum thunbergii (Mertens) O. Kuntze in Maizuru Bay, Japan Sea. Botanical Magazine 87:285-292.

UMEZAKI, I. 1984. Ecological studies of Sargassum horneri (Turner) C. Agardh in Obama Bay, Japan Sea. Bulletin of the Japanese Society of Scientific Fisheries 50:1193-1200.

UNDERWOOD, A.J., KINGSFORD, M.J. \& ANDREW, N.L. 1991. Patterns in shallow subtidal marine assemblages along the coast of New South Wales. Australian Journal of Ecology 6:231-249.

WANDERS, J.B.W. 1976. The role of benthic algae in the shallow reef of Curaçao (Netherlands Antilles). II - Primary productivity of the Sargassum beds on the north-east coast submarine plateau. Aquatic Botany 2:327-335.

YONESHIGUE, Y. \& VALENTIN, J.L. 1988. Comunidades algais fotófilas de infralitoral de Cabo Frio, Rio de Janeiro, Brasil. Gayana, série Botanica, 45:61-75.

ZAR, J.H. 1984. Biostatistical analysis. 2 ed. Prentice-Hall, New Jersey. 